\title{
New approach to numerical computation of the eigenfunctions of the continuous spectrum of three-particle Schrödinger operator. I. One-dimensional particles, short-range pair potentials
}

\author{
V. S. Buslaev ${ }^{1}$, S. B. Levin ${ }^{1}$, P. Neittaanmäki², T. Ojala ${ }^{2}$ \\ ${ }^{1}$ Department of Mathematical and Computational Physics, \\ St-Petersburg State University, Russia \\ ${ }^{2}$ Department of Mathematical Information Technology, \\ University of Jyvaskyla, Finland
}

\begin{abstract}
Basing on analogy between the three-body scattering problem and the diffraction problem of the plane wave (for the case of the short range pair potentials) by the system of six half transparent screens, we presented a new approach to the few-body scattering problem. The numerical results have been obtained for the case of the short range nonnegative pair potentials. The presented method allows a natural generalization to the case of the long range pair potentials.
\end{abstract}

\section{Introduction}

\section{1}

The quantum system of two particles interacting via the Coulomb potential is probably the most known model of the Quantum mechanics. The model allows an explicit solution. Oppositely, the mathematical status of the system of three quantum particles with the pair Coulomb interaction is relatively poor. The system of three particles with short range pair interactions was successfully studied by L. Faddeev [1], but the direct generalization to the Coulomb type potentials was found impossible. Something, however, is known: the quantative nature of the spectrum and the asymptotic behavior of the solutions of the non-stationary Schrödinger equation. These results were obtained in frameworks of a non-stationary approach, see [2, 3. Nevertheless a mathematically consistent stationary approach similar to the Lippmann-Schwinger integral equation, or something analogous, was not developed though. Such an approach is needed if we are interested in numerical parameters of many important physical processes like dissociative recombination in atomic and molecular physics with applications to astrophysics, formation and break up processes of large molecules in bioengineering and medicine, formation of the molecular resonance states in chemical physics, dynamics of the few electron systems in wave-conductor nano-technology. 
There are specific difficulties that are characteristic for the systems with Coulomb type interactions.

They are naturally explained by the fact that the long range interactions crucially affects the asymptotic behavior at infinity in the configuration space of the eigenfunctions, Green's functions and other similar objects. The consequences of that affection on the structure of asymptotics up to now have not been taken in account in correct mathematical manner. As a result, such approaches to many particle scattering as Faddeev's equations [1], AGS equations [5], successfully applicable to the systems with short range potentials, do not work for the systems with the long range potentials.

The asymptotic behavior of the wave functions for the systems of few charged particles has been studied only in some domains of configuration space but not for all asymptotic directions. Let us shortly list some known results. In [6, 7] there was studied the asymptotic behavior of three charged particles wave function for the case of large distances between all three particles. Another limiting case, considered in [8], corresponds to configurations with one Jacobi coordinates been much larger than another one.

In the list of the literature reflecting the theoretical aspects of the problem we mention also [10], 11], 12, [13, [14, 15, 16]. Application to computational aspects of the problem were treated in [17, 18, 19, 20, 21, 22], 23].

One of the typical computational approaches to such systems is to replace the Coulomb potentials by the Yukava potentials (or some other cut-off potentials), to compute the parameters of the scattering for this modified system, and to consider the results for small screening parameter. Mathematically, it is not a completely satisfactory procedure. Some other approximate approaches also exist.

\section{2}

We started a description of a new approach to the mathematically consistent stationary treatment of the scattering in the systems of three quantum particles with long range pair interactions in [27]. We are going to consider in turn the case of three onedimensional particles with short range interaction (it is already completed theoretically and published), the case of three one-dimensional particles with long range (Coulomb type) interactions (it is also completed at the moment but is not published yet) and the case of three three-dimensional particles. Each next case will be based on the results for the preceding stage. We hope that we will be able to illustrate each theoretical stage by numerical computation of the field. This paper contains the numerical results illustrating the formulas of paper [27].

We assume here that the pair potentials are non-negative. In this case the spectrum is purely continuous, covers the positive semi-axis and is in the natural sense homogeneous. In fact, this case is the most interesting at the present stage since the lower spectral branches for negative total energy in case of charged particles was already treated in [28.

It is worth mentioning that the scattering in the system of one-dimensional particles is not just a first step on the way to the case of three-dimensional particles. It is interesting by itself, the systems of three one-dimensional particles (neutral or charged) were intensively studied during many years (see, for example, [29, 30, 31, 32, 33]). In recent years there appeared a new interest to such systems since they were realized experimentally (see [34, 35, 36, 37]).

The main idea is to suggest a priori explicit formulas for the asymptotic behavior of the eigenfunctions of the continuous spectrum (for example, the scattered plane 
waves).

The formulas describe the eigenfunctions at infinity up to the simple diverging waves with smooth amplitudes. If we are able to find such asymptotic behavior (satisfying certain criteria that will be discussed later on) even heuristically, we obtain a way for regular numerical computations of the eigenfunctions. We obtain simultaneously also a method to construct an appropriate integral equation of the same nature as the Lippmann-Schwinger equation for the scattering of the plane wave by a quickly decreasing potential that can be used to justify the asymptotic behavior rigorously following the ideas of [4].

For one-dimensional particles with quickly decreasing at infinity pair potentials we can use, for the description of the mentioned asymptotic behavior, the analogy between the stated problem and the classical problem of the diffraction of the plane waves by the set of semi-transparent infinite screens. This analogy was already used in $[24,25,26,27$. In case of long range potentials we are able to treat the diffraction problem analogously with the replacement of the classical plane waves by plane waves that are appropriately deformed by the long range tails of the Coulomb potentials. It is important to mention that the diffraction itself and the corresponding scattering problems cannot be completely reduced to the scattering of the plane waves by the screens; we have to add to these processes some genuine diffraction components that have more complicated analytical structure but still explicit description. This more complicated structure is also dictated by the analogy with the classical diffraction theory.

Here we consider a system of three identical one-dimensional quantum particles interacting via short-range pair potentials. These strict limitations allow to simplify the narration and the view of the formulas, but the essence of the main questions which we are interested in and their treatments is not affected. In the following parts we consequentially will get rid of these limitations. As we have mentioned above, the theoretical part of this work is already published, but we decided for the completeness to repeat shortly the main theoretical ideas of [27]. The main goal of the work is to confirm that the approach works for the numerical computation of the eigenfunctions of the continuous spectrum. The approach is new even for the short range pair potentials.

The structure of the work is as following: it consists of two parts. The first part is devoted to the known theoretical constructions. The second one is original and represents the results of numerical computer computations.

\section{Main formulas}

\subsection{Configuration plane}

The configuration space of the system after the separation of motion of the center of mass is the hyperplane $\Gamma=\left\{\mathbf{x}=\left(x_{1}, x_{2}, x_{3}\right): x_{1}+x_{2}+x_{3}=0\right\}$ in $\mathbf{R}^{3}$. The Schroedinger equation has the form:

$$
-\triangle \psi+\left(v\left(x_{1}\right)+v\left(x_{2}\right)+v\left(x_{3}\right)\right) \psi=E \psi,
$$

where $\psi=\psi(\mathbf{x}) \in \mathbf{C}, \triangle$ is the Laplace operator on $\Gamma$ that will be described more specifically later on. The real-valued function $v(x), x \in \mathbf{R}$, is the potential of the pair interaction. In the present text it is supposed to be an even function with a compact support, $v(x)=0,|x|>b / 2$. We suppose $E>0$. 
The scalar product on $\Gamma$ is given by the formula

$$
<\mathbf{x}, \mathbf{x}^{\prime}>=\frac{2}{3}\left(x_{1} x_{1}^{\prime}+x_{2} x_{2}^{\prime}+x_{3} x_{3}^{\prime}\right) .
$$

As usual, the norm of the vector is defined by the formula $|\mathbf{x}|^{2}=<\mathbf{x}, \mathbf{x}>$. The Laplacian is also generated by this scalar product.

Let us consider on $\Gamma$ three straight lines $l_{j}=\left\{\mathbf{x}: x_{j}=0\right\}, j=1,2,3$, and three unit vectors $\mathbf{l}_{j}$ that belong to these lines and oriented such that $x_{j+1}$ increases along $\mathbf{l}_{j}$. Consider also the unit vectors $\mathbf{k}_{j}$ that are orthogonal to $\mathbf{l}_{j}$ and oriented along the direction of increasing of $x_{j}$. Consider, at last, three pairs of the cartesian coordinates $\left(x_{j}, y_{j}\right)$ with respect to the bases $\left(\mathbf{k}_{j}, \mathbf{l}_{j}\right)$. These are, so called, Jacobian coordinates on $\Gamma$. With these coordinates

$$
<\mathbf{x}, \mathbf{x}^{\prime}>=x_{j} x_{j}^{\prime}+y_{j} y_{j}^{\prime}, \quad|\mathbf{x}|^{2}=x_{j}^{2}+y_{j}^{2}, \quad j=1,2,3,
$$

and

$$
\triangle=\frac{\partial^{2}}{\partial x_{j}^{2}}+\frac{\partial^{2}}{\partial y_{j}^{2}}, \quad j=1,2,3 .
$$

The lines $l_{j}$ define on the plane $\Gamma$ six sectors. The internal part of a certain one consists of the vectors $\left(x_{1}, x_{2}, x_{3}\right)$ whose coordinates satisfy the condition $x_{j_{1}}>x_{j_{2}}>$ $x_{j_{3}}$ where $\sigma=\left(j_{1}, j_{2}, j_{3}\right)$ is a permutation of the numbers (123). We will denote any sector by the corresponding permutation $\sigma$ and will write $\lambda=\lambda_{\sigma}$ (see Figure 1).

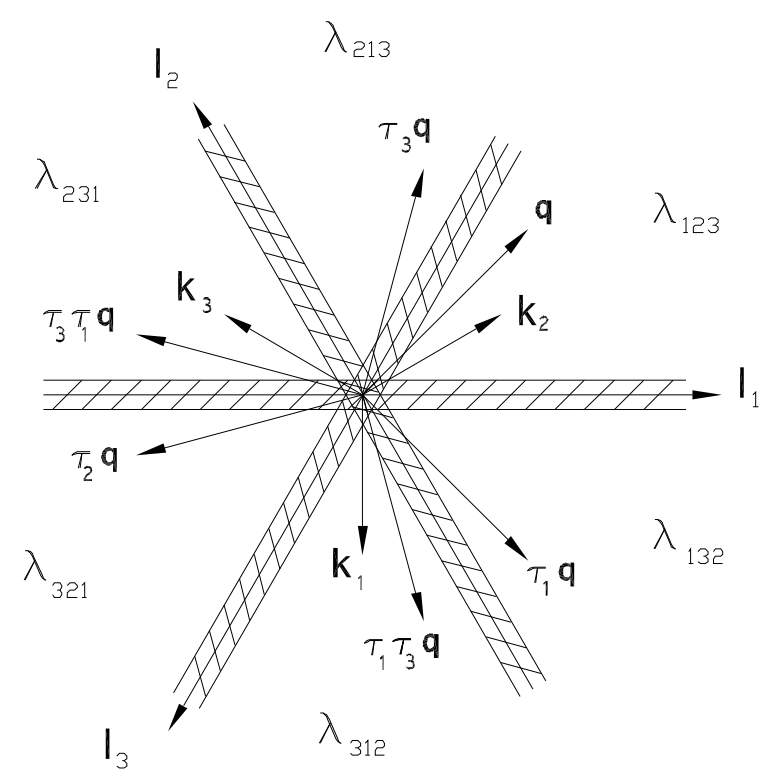

Figure 1

Let the group $S_{3}$ of permutation acts on $\Gamma$ so that

$$
(\sigma, \mathbf{x}) \rightarrow \sigma \mathbf{x}=\left(x_{j_{1}}, x_{j_{2}}, x_{j_{3}}\right), \quad \sigma=\left(j_{1}, j_{2}, j_{3}\right), \quad \mathbf{x}=\left(x_{1}, x_{2}, x_{3}\right) .
$$


The group contains 6 elements. The permutation can be identical, or a transposition of two elements, or a composition of two transpositions, some of the compositions coincide. Introduce the notations for the transpositions: $\tau_{1}=(132), \tau_{2}=(321)$, $\tau_{3}=(213)$, and notice that $\tau_{i}^{2}=I, \quad i=1,2,3$. The action of the transposition on $\Gamma$ will be denoted by the same symbol $\tau_{j}, \quad j=1,2,3$. It corresponds to the reflection with respect to the line $l_{j}, j=1,2,3$. It is clear that

$$
\tau_{1}\left(x_{1}, x_{2}, x_{3}\right)=\left(-x_{1},-x_{3},-x_{2}\right), \quad \tau_{1}\left(y_{1}, y_{2}, y_{3}\right)=\left(y_{1}, y_{3}, y_{2}\right),
$$

the analogous formulas are also satisfied for $\tau_{2}, \tau_{3}$. The composition of two transpositions generates a rotation, and the following equalities, in particular, hold: $\tau_{1} \tau_{2}=$ $\tau_{3} \tau_{1}=\tau_{2} \tau_{3}, \tau_{2} \tau_{1}=\tau_{1} \tau_{3}=\tau_{3} \tau_{2}$

Six elements $\tau$ of the group $S_{3}$ generate six vectors $\tau \mathbf{q}$. If $\mathbf{q} \in \lambda_{\sigma}$ then $\tau \mathbf{q} \in \lambda_{\tau \sigma}$.

\subsection{Separation of variables}

Consider now the eigenfunction that describes the scattering in the system where just one of three potentials is not equal to zero. Now we deal with the Schrödinger equation

$$
-\triangle \chi_{j}+v\left(x_{j}\right) \chi_{j}=E \chi_{j}
$$

It allows the separation of variables:

$$
\chi_{j}(\mathbf{x}, \mathbf{q})=\chi\left(x_{j}, k_{j}\right) e^{i p_{j} y_{j}} .
$$

The sense of the variables $\left(x_{j}, y_{j}\right)$ is clear, $\left(k_{j}, p_{j}\right)$ are the Jacobian coordinates of a given vector $\mathbf{q}$.

The function $\chi(x, k), x, k \in \mathbf{R}$, is a solution of the ordinary differential equation

$$
-\chi_{x x}+v(x) \chi=k^{2} \chi
$$

it has to be described separately. For $k>0$ there exists and is unique the solution that is characterized by the following asymptotic behavior:

$$
\chi(x, k) \sim s(k) e^{i k x}, x \rightarrow+\infty ; \quad \chi(x, k) \sim e^{i k x}+r(k) e^{-i k x}, x \rightarrow-\infty .
$$

On the whole axis $k$ this solution, due to the evenness of the potential, has to be extended by the formula $\chi(x, k)=\chi(-x,-k)$. Here $s$ and $r$ are some complex-valued functions of $k$ that are called the transition and the reflection coefficients.

We will suppose here that $v(x) \geq 0$ therefore the equation (88) does not have the bound states.

\subsection{Formal setting of the problem}

Our final goal is to construct the solution $\psi(\mathbf{x}, \mathbf{q})$ of the Schrödinger equation that is characterized by the following behavior at infinity:

$$
\psi=n(\hat{\mathbf{x}}, \mathbf{q}) \frac{e^{-i|\mathbf{x}||\mathbf{q}|}}{|\mathbf{x}|^{1 / 2}}+f(\hat{\mathbf{x}}, \mathbf{q}) \frac{e^{i|\mathbf{x}||\mathbf{q}|}}{|\mathbf{x}|^{1 / 2}}+o\left(\frac{1}{|\mathbf{x}|^{1 / 2}}\right), \quad \hat{\mathbf{x}}=\frac{\mathbf{x}}{|\mathbf{x}|} .
$$

Here

$$
n(\hat{\mathbf{x}}, \mathbf{q})=\sqrt{\frac{2 \pi}{i|\mathbf{q}|}} \delta(\hat{\mathbf{x}}, \hat{\mathbf{q}})
$$


and the $\delta$ function has to be considered with respect to the angle measure on the unit circle.

The asymptotic behavior has to be treated in a weak sense (in sense of distributions) with respect to $\hat{\mathbf{x}}$. The coefficient $n$ before the converging circle wave coincides with the analogous coefficient before the converging wave in the weak asymptotic representation of the plane wave $e^{i<\mathbf{x}, \mathbf{q}>}$. Therefore the solution $\psi(\mathbf{x}, \mathbf{q})$ can be naturally called the scattered plane wave.

Due to the symmetries of the potential $\psi(\mathbf{x}, \mathbf{q})=\psi(\sigma \mathbf{x}, \sigma \mathbf{q}), \sigma \in S$, we always can assume that $\mathbf{q}$ belongs to a certain sector, say $\lambda_{I} \equiv \lambda_{123}$. We restrict ourselves here by the assumption that $\mathbf{q}$ does not belong to neighborhoods of the boundaries of the sector. It would be not hard to consider also the case when $\mathbf{q}$ belongs to the lines $l_{j}$ and their neighborhoods.

The function $f$ is a singular distribution. We will see that it has singularities on all six directions $\sigma \mathbf{q}, \sigma \in S$. Four of them are of $\delta$ - function type, two (for $\left.\sigma \mathbf{q}=\tau_{2} \tau_{3} \mathbf{q}, \tau_{2} \tau_{1} \mathbf{q}\right)$ are of type of Cauchy's limiting kernel. It is worth to notice that although the asymptotic behavior is singular the solution itself is, naturally, a smooth function.

In the case of the scattering by a quickly decreasing at infinity potential the asymptotic behavior is given by the formula

$$
\psi(\mathbf{x}, \mathbf{q})=e^{i<\mathbf{x}, \mathbf{q}>}+f(\hat{\mathbf{x}}, \mathbf{q}) \frac{e^{i|\mathbf{x}||\mathbf{q}|}}{|\mathbf{x}|^{1 / 2}}+o\left(\frac{1}{|\mathbf{x}|^{1 / 2}}\right),
$$

where that time the scattering amplitude $f$ is not a singular distribution, but a smooth function, and the asymptotic behavior can be treated in uniform sense.

Under our assumptions over the potential the scattered plane waves create for $E>0$ a complete system of the eigenfunctions of the uniform in multiplicity continuous spectrum of the three particle Schrödinger operator, $E \geq 0$.

Our further plan is following: we construct in explicit form a function $\psi_{1}(\mathbf{x}, \mathbf{q})$ and hope that the difference $\psi-\psi_{1}$ has the diverging asymptotic behavior

$$
\psi(\mathbf{x}, \mathbf{q})-\psi_{1}(\mathbf{x}, \mathbf{q})=g(\hat{\mathbf{x}}, \mathbf{q}) \frac{e^{i|\mathbf{x}||\mathbf{q}|}}{|\mathbf{x}|^{1 / 2}}+o\left(\frac{1}{|\mathbf{x}|^{1 / 2}}\right)
$$

where $g$ is a continuous function of the arguments.

Constructing $\psi_{1}$ we use two criteria: (1) The discrepancy

$$
Q\left[\psi_{1}\right](\mathbf{x}, \mathbf{q})=-\triangle \psi_{1}+\left(v\left(x_{1}\right)+v\left(x_{2}\right)+v\left(x_{3}\right)\right) \psi_{1}-E \psi_{1}, \quad E=|\mathbf{q}|^{2} .
$$

sufficiently quickly vanishes at infinity, (2) The asymptotic representation for $\psi_{1}-$ $e^{i<\mathbf{x}, \mathbf{q}>}$ contains asymptotically only the diverging wave.

Consider the difference

$$
\xi=\psi-\psi_{1}
$$

It satisfies the equation

$$
H \xi-E \xi=-Q, \quad H=-\triangle+\left(v\left(x_{1}\right)+v\left(x_{2}\right)+v\left(x_{3}\right)\right) .
$$

Since $Q$ is quickly vanishing one can hope that $\xi$ asymptotically behaves as the diverging wave

$$
\xi(\mathbf{x}, \mathbf{q})=g(\widehat{\mathbf{x}}, \mathbf{q}) \frac{e^{i|\mathbf{q}||\mathbf{x}|}}{|\mathbf{x}|^{1 / 2}}+o\left(\frac{1}{|\mathbf{x}|^{1 / 2}}\right)
$$


with a continuous amplitude $g$. In other words, $\xi$ satisfies the classical radiation conditions at infinity.

Further, it is naturally to hope that for $\xi$ we can construct an integral equation with the same properties as the properties of classical Lippmann-Schwinger equation. We can do it developing the ideas of work [4]. However, preliminary, we can try to use (16)-(17) for the numerical computation of $\xi$ and, consequently, of $\psi$. For the numerical computations we can replace (17) by approximate boundary condition

$$
\left(\frac{\partial}{\partial|x|}-i \sqrt{E}\right) \xi=0, \text { for }|x|=R
$$

where $R$ is sufficiently large.

The following construction of $\psi_{1}$ will consist of two steps. At first, we construct for $\psi_{1}$ so called ray approximation $\psi_{R}$. Its discrepancy has some singularities. After a natural modification motivated by some classical diffraction problems the discrepancy will become a smooth function.

\subsection{Ray approximation}

Consider six vectors $\sigma \mathbf{q}$. These vectors, more precisely, spanned by them rays, separate six sectors that we denote $K_{j}^{ \pm}$. The indices of the notation coincide with the indices of the vector $\pm \mathbf{l}_{j}$ that belongs to the sector $K_{j}^{ \pm}$. $K_{j}^{ \pm}$

Now we can give explicit expressions for the ray approximation in different sectors $\underline{\text { Sector } K_{1}^{+}:} \psi_{R}=\psi_{1}^{+}$,

$$
\psi_{1}^{+}(\mathbf{x}, \mathbf{q})=\chi_{1}(\mathbf{x}, \mathbf{q}) s_{2} s_{3} .
$$

We use here the following notations: $s_{j}=s\left(k_{j}\right), r_{j}=r\left(k_{j}\right)$.

Sector $K_{3}^{-}: \psi_{R}=\psi_{3}^{-}$,

$$
\psi_{3}^{-}(\mathbf{x}, \mathbf{q})=\chi_{3}(\mathbf{x}, \mathbf{q}) s_{1} s_{2}
$$

$\underline{\text { Sector } K_{2}^{+}:} \psi_{R}=\psi_{2}^{+}$,

$$
\psi_{2}^{+}(\mathbf{x}, \mathbf{q})=\chi_{2}(\mathbf{x}, \mathbf{q}) s_{1}+\chi_{2}\left(\mathbf{x}, \tau_{3} \mathbf{q}\right) s_{2} r_{3} .
$$

Sector $K_{2}^{-}: \psi_{R}=\psi_{2}^{-}$,

$$
\psi_{2}^{-}(\mathbf{x}, \mathbf{q})=\chi_{2}(\mathbf{x}, \mathbf{q}) s_{3}+\chi_{2}\left(\mathbf{x}, \tau_{1} \mathbf{q}\right) s_{2} r_{1}
$$

Sector $K_{1}^{-}: \psi_{R}=\psi_{1}^{-}$,

$$
\psi_{1}^{-}(\mathbf{x}, \mathbf{q})=\chi_{1}(\mathbf{x}, \mathbf{q})+\chi_{1}\left(\mathbf{x}, \tau_{2} \mathbf{q}\right) r_{2} s_{1}+\chi_{1}\left(\mathbf{x}, \tau_{3} \tau_{1} \mathbf{q}\right) r_{2} r_{1}+\chi_{1}\left(\mathbf{x}, \tau_{3} \mathbf{q}\right) r_{3} .
$$

Sector $K_{3}^{+}: \psi_{R}=\psi_{3}^{+}$,

$$
\psi_{3}^{+}(\mathbf{x}, \mathbf{q})=\chi_{3}(\mathbf{x}, \mathbf{q})+\chi_{3}\left(\mathbf{x}, \tau_{2} \mathbf{q}\right) r_{2} s_{3}+\chi_{3}\left(\mathbf{x}, \tau_{1} \tau_{3} \mathbf{q}\right) r_{2} r_{3}+\chi_{3}\left(\mathbf{x}, \tau_{1} \mathbf{q}\right) r_{1}
$$

The total field $\psi_{R}$ is defined by the formula

$$
\psi_{R}=\theta_{1}^{+} \psi_{1}^{+}+\theta_{3}^{-} \psi_{3}^{-}+\theta_{2}^{+} \psi_{2}^{+}+\theta_{2}^{-} \psi_{2}^{-}+\theta_{1}^{-} \psi_{1}^{-}+\theta_{3}^{+} \psi_{3}^{+} .
$$

The notation $\theta_{j}^{( \pm)}$is used here for the characteristic function of the corresponding sector $K_{j}^{ \pm}$,

$$
\theta_{1}^{+}+\theta_{3}^{-}+\theta_{2}^{+}+\theta_{2}^{-}+\theta_{1}^{-}+\theta_{3}^{+}=1
$$


In this formula the value of the field $\psi_{R}$ on the boundaries of the sectors is not defined. In [27] it was shown that on all boundary rays except two, directed along the vectors

$$
\mathbf{q}_{23} \equiv \tau_{2} \tau_{3} \mathbf{q}, \quad \mathbf{q}_{21} \equiv \tau_{2} \tau_{1} \mathbf{q},
$$

the field is smooth, and its discrepancy everywhere except the two vectors is equal to zero.

\subsection{Diffraction corrections}

The diffraction corrections on rays directed along the vectors $\mathbf{q}_{23}$ and $\mathbf{q}_{21}$, can be constructed quite easily. Consider the sector $\lambda_{231}$ containing $\mathbf{q}_{23}$. Introduce the polar coordinates $(r=|\mathbf{x}|, \omega)$. Let us orient the angle from $\mathbf{l}_{2}^{+}$to $\mathbf{l}_{1}^{-}$. Let $\omega_{23}$ correspond to $\mathbf{q}_{23}$. Introduce four angles $0<\omega_{1}<\omega_{2}<\omega_{23}<\omega_{3}<\omega_{4}<\pi / 3$. Consider the open covering of the interval $(0, \pi / 3)$ by the subintervals $\left(0, \omega_{2}\right),\left(\omega_{1}, \omega_{4}\right),\left(\omega_{3}, \pi / 3\right)$ and introduce a subordinated partition of unit:

$$
1=\zeta_{1}+\zeta_{2}+\zeta_{3}
$$

Further consider the function

$$
\Phi(\alpha)=\frac{e^{-i \frac{\pi}{4}}}{\sqrt{\pi}} \int_{\infty}^{\alpha} e^{i t^{2}} d t .
$$

Notice that

$$
\Phi(\alpha) \rightarrow 1, \text { as } \alpha \rightarrow+\infty, \quad \Phi(\alpha) \rightarrow 0, \text { as } \alpha \rightarrow-\infty .
$$

In more detail:

$$
\Phi(\alpha)=1+\frac{e^{-i \frac{\pi}{4}}}{\sqrt{\pi}} \frac{e^{i \alpha^{2}}}{2 i \alpha}+\Delta \Phi(\alpha), \quad \Delta \Phi(\alpha)=-\frac{e^{-i \frac{\pi}{4}}}{\sqrt{\pi}} \int_{\alpha}^{\infty} \frac{e^{i t^{2}}}{2 i t^{2}} d t=O\left(\alpha^{-3}\right), \text { when } \alpha \rightarrow+\infty .
$$

Introduce the function

$$
\begin{aligned}
& \Phi_{1}^{(23)}=\Phi\left(\operatorname{sign}\left(\omega_{23}-\omega\right)|| \mathbf{q}_{23}|| \mathbf{x}\left|-<\mathbf{q}_{23}, \mathbf{x}>\right|^{1 / 2}\right), \\
& \Phi_{2}^{(23)}=\Phi\left(\operatorname{sign}\left(\omega-\omega_{23}\right)|| \mathbf{q}_{23}|| \mathbf{x}\left|-<\mathbf{q}_{23}, \mathbf{x}>\right|^{1 / 2}\right) .
\end{aligned}
$$

It is known that

$$
\phi=e^{i<\mathbf{x}, \mathbf{q}_{23}>} \Phi_{j}^{(23)}
$$

satisfies the Helmholtz equation $-\triangle \phi-E \phi=0$.

Now we can describe the diffraction corrections to the ray approximation on $\lambda_{231}$. For that the ray field $\psi_{R}=\theta_{2}^{+} \psi_{2}^{+}+\theta_{1}^{-} \psi_{1}^{-}$in the sector $\lambda_{231}$ is replaced by

$$
\psi_{D}^{(23)}=\psi_{R}+\zeta_{2} e^{i<\mathbf{q}_{23}, \mathbf{x}>}\left[R_{1}\left(\Phi_{1}^{(23)}-\theta_{2}^{+}\right)+R_{2}\left(\Phi_{2}^{(23)}-\theta_{1}^{-}\right)\right] .
$$

$R_{1}=r_{1} s_{2} r_{3}, R_{2}=r_{3} r_{2} s_{1}+s_{3} r_{2} r_{1}$.

Notice that the field $\psi_{R}$ on the interval $\left(\omega_{1}, \omega_{4}\right)$ contains the discontinuous component

$$
\psi_{J}=e^{i<\mathbf{q}_{23}, \mathbf{x}>}\left[\theta_{2}^{+} R_{1}+\theta_{1}^{-} R_{2}\right],
$$

so the sense of the modification in nothing else but a simple replacement of this discontinuous on $\mathbf{q}_{23}$ component by a smooth solution of the Helmholtz equation that outside of $\left(\omega_{2}, \omega_{3}\right)$ gradually transfers to the original discontinuous component up to 
a diverging circle wave with a smooth amplitude. Outside of the interval $\left(\omega_{1}, \omega_{4}\right)$ the function $\psi_{D}$ coincides with the original ray approximation $\psi_{R}$.

Analogous constructions can be also considered in the sector $\lambda_{312}$. It is also worth to introduce here the polar coordinates, and again to suppose that the angle $\omega$ varies in the same limits with the same orientation, from $\mathbf{l}_{3}$ to $-\mathbf{l}_{2}$. We again can introduce the angles $\omega_{21}, \omega_{j}, j=1,2,3,4$ and a cutoff function $\zeta_{2}$. After that the modified field on $\lambda_{312}$ can be described by the formula

$$
\psi_{D}^{(21)}=\psi_{R}+\zeta_{2} e^{i<\mathbf{q}_{21}, \mathbf{x}>}\left[R_{2}\left(\Phi_{1}^{(21)}-\theta_{3}^{+}\right)+R_{1}\left(\Phi_{2}^{(21)}-\theta_{2}^{-}\right)\right] .
$$

Here

$$
\begin{aligned}
& \Phi_{1}^{(21)}=\Phi\left(\operatorname{sign}\left(\omega_{21}-\omega\right)|| \mathbf{q}_{21}|| \mathbf{x}\left|-<\mathbf{q}_{21}, \mathbf{x}>\right|^{1 / 2}\right), \\
& \Phi_{2}^{(21)}=\Phi\left(\operatorname{sign}\left(\omega-\omega_{21}\right)|| \mathbf{q}_{21}|| \mathbf{x}\left|-<\mathbf{q}_{21}, \mathbf{x}>\right|^{1 / 2}\right) .
\end{aligned}
$$

As a result everywhere on $\Gamma$ outside of some circle $C_{r_{1}}$ with the center at 0 and the radius $r_{1}$ there appears a smooth approximate wave field $\psi_{0}$ :

$$
\psi_{0}=\psi_{R} \theta_{I}+\psi_{D}^{(23)} \theta_{231}+\psi_{D}^{(21)} \theta_{312}
$$

Here $\theta_{231}$ and $\theta_{312}$ are the characteristic functions of the corresponding $\lambda$-sectors, and $\theta_{I}$ is the characteristic function of their complement. Again there are no jumps on the boundaries of the $\lambda$-sectors.

Consider a circle with the center at the origin. The radius $r_{1}$ of this circle is defined by the condition that outside of the circle on the rays directed along the vectors $\sigma \mathbf{q}$ the sum of the pair potentials is equal to zero. Under this condition the field $\psi_{0}$ can be additionally modified with the help of the cutoff function $\zeta(|\mathbf{x}|)$ that is equal to 0 for $|\mathbf{x}|<r_{1}$ and to 1 for $|\mathbf{x}|>r_{2}$ where $r_{1}<r_{2}$.

The final expression for the approximate field is now

$$
\psi_{1}=\psi_{0} \zeta
$$

\subsection{Discrepancy}

We remember that there were proposed two criteria that have to be taken into account when constructing the function $\psi_{1}$. It is sufficiently clear that the second one : (2) The difference $\psi_{1}-e^{i<\mathbf{x}, \mathbf{q}>}$ contains asymptotically (in the weak sense) only the diverging circle wave, is fulfilled. It is remained to check the first one: (1) The discrepancy

$$
Q\left[\psi_{1}\right](\mathbf{x}, \mathbf{q})=-\triangle \psi_{1}+\left(v\left(x_{1}\right)+v\left(x_{2}\right)+v\left(x_{3}\right)\right) \psi_{1}-E \psi_{1}, \quad E=|\mathbf{q}|^{2} .
$$

sufficiently quickly vanishes at infinity.

From the previous formulas it follows that outside a certain circle of the radius $r_{1}$ the discrepancy is not equal to zero only on some neighborhoods the rays generated by the vectors $\mathbf{q}_{23}$ and $\mathbf{q}_{21}$. On these neighborhoods the discrepancy vanishes as $|\mathbf{x}|^{-5 / 2}$. It follows from this that the relative scattering amplitude $g(\hat{\mathbf{x}}, \mathbf{q})$, see (13), must be continuous. Here we give for the discrepancy a formula that can be used for the numerical computations of $\psi$.

Consider now the field $\psi_{1}$ on the neighborhoods of $\mathbf{q}_{23}$ and $\mathbf{q}_{21}$. It is not hard to see that the discrepancy of this expression is equal to zero on the sectors where there is equal to zero the derivative of the function $\zeta_{2}$. It means that the discrepancy $Q\left[\psi_{0}\right]$ 
can differ from zero only on the subintervals $\left(\omega_{1}, \omega_{2}\right)$ and $\left(\omega_{3}, \omega_{4}\right)$. That implies that the discrepancy $Q^{(23)}$ on the sector $\lambda_{231}$ can be naturally represented as the sum:

$$
Q^{(23)}=Q_{1}^{(23)}+Q_{2}^{(23)} .
$$

Similarly, on the sector $\lambda_{312}$

$$
Q^{(21)}=Q_{1}^{(21)}+Q_{2}^{(21)}
$$

All four terms here can be easily computed. The answers are completely analogous. In particular,

$$
\begin{gathered}
Q_{1}^{(23)}=R_{1}(-\Delta-E) e^{i<\mathbf{q}_{23}, \mathbf{x}>}\left(\Phi_{1}^{(23)}-1\right) \zeta_{2}^{\prime}= \\
=R_{1}\left[e^{i<\mathbf{q}_{23}, \mathbf{x}>}\left(\Phi_{1}^{(23)}-1\right) \frac{-1}{r^{2}} \zeta_{2}^{\prime \prime}-2 i \frac{1}{r} \zeta_{2}^{\prime}<\mathbf{q}_{23}, w>e^{i<\mathbf{q}_{23}, \mathbf{x}>} \Delta \Phi_{1}^{(23)}\right] .
\end{gathered}
$$

where $w$ is a unit vector orthogonal to $\hat{\mathbf{x}}$ and oriented along the direction of increasing $\omega$.

Finally,

$$
Q\left[\psi_{0}\right]=Q_{1}^{(23)}+Q_{2}^{(23)}+Q_{1}^{(21)}+Q_{2}^{(21)} .
$$

It is easy to see that all four components of the discrepancy vanish at infinity like $|\mathbf{x}|^{-5 / 2}$.

The previous computations of the discrepancy were given for not small $|\mathbf{x}|$ where the supports of three potentials are separated. Let us modify now the field $\psi_{1}$ by introducing in it the factor $\zeta=\zeta(|\mathbf{x}|)$ that is equal to 0 for $|\mathbf{x}|<r_{1}$, and is equal to 1 for $|\mathbf{x}|>r_{2}, 0<r_{1}<r_{2}$. It is supposed that for $|\mathbf{x}|>r_{1}$ three supports do not intersect. The definition of $\psi_{1}$ is given by the formula

$$
\psi_{1}=\psi_{0} \zeta
$$

The final expression for the discrepancy is given by the formula

$$
Q\left[\psi_{1}\right]=Q\left[\psi_{0}\right] \zeta-2\left[\frac{\partial}{\partial|\mathbf{x}|} \psi_{0}(\mathbf{x}, \mathbf{q})\right] \zeta^{\prime}-\psi_{0} \frac{1}{|\mathbf{x}|} \frac{\partial}{\partial|\mathbf{x}|}|\mathbf{x}| \frac{\partial}{\partial|\mathbf{x}|} \zeta .
$$

There is no problem in explicit computation of the derivative $\frac{\partial}{\partial|\mathbf{x}|} \psi_{0}(\mathbf{x}, \mathbf{q})$.

\section{Numerical computations}

The goal of the computations was to show that the suggested plan is realistic and can be practically used for the computations of the scattered plane wave and the corresponding amplitude of scattering. The pair-particle potential $v(x)$ and the vector $\mathbf{q}$ are two parameters of the problem.

As for $v(x)$ we choose the potential function

$$
v(x)=\left\{\begin{array}{lc}
2 e^{\frac{1}{(4 x)^{2}-1}+1}, & |x|<\frac{1}{4} \\
0, & \text { otherwise. }
\end{array}\right.
$$

Any specific choice is not crucial, we could take arbitrary even potential (even nonnecessary continuous) with the compact support. With this potential we computed the solution $\chi(x, k)$ of one-dimensional Schrödinger equation (6) and found the corresponding transition $s(k)$ and reflection $r(k)$ coefficients. 
Then the solutions $\chi(x, k)$ were interpolated to the actual computational domain to construct the functions $\chi_{j}$. This interpolation was necessary only on the support of the potentials. Outside of the supports the analytic expressions of the functions $\chi(x, k)$ were known after the coefficients $s(k)$ and $r(k)$ were found numerically.

We took $E=4$. For the vector $\mathbf{q}$ we used two choices: 1) $k_{1}=1, p_{1}=\sqrt{3}, 2$ ) $k_{1}=p_{1}=\sqrt{2}$. In the first case the field as a function of $\mathbf{x}$ is symmetric with respect to the straight line generated by $\mathbf{q}$. It was taken for the control.

The function $\psi_{R}$ was computed directly with the knowledge of $\chi_{j}$, the Fresnel integral was taken from GSL (Gnu scientific library).

The functions $\psi_{0}(\mathbf{x}, \mathbf{q})$ and $\psi_{1}(\mathbf{x}, \mathbf{q})$ were computed with the help of the explicit formulas for them. The discrepancy $Q$ was also computed with the help of the explicit formulas. The radii $r_{1}<r_{2}$ were taken as $r_{1}=4, r_{2}=14.5$. We think that this choice reasonably corresponds to the selected value of $|\mathbf{q}|$.

For the diffraction corrections (and near the origin) the chosen partition of unity corresponds to function $\zeta(z)=z^{3}\left(10-15 z+6 z^{2}\right), 0<z<1$, where $z$ is the variable relative to angle $\omega$.

Then we finally considered the boundary problem (16-18). Of course, it was the main part of the numerical program of the work. The problem on the disc is not on the spectrum.

For the computations we used mainly FreeFem ++ , which is a user friendly language dedicated for solving partial differential equations with the finite element method. All the necessary steps from mesh creation to solving the linear system can be done within the same program in a manner that is not of a black box type. Since we used the finite element method, we introduced the corresponding weak formulation of the problem: find $\xi \in H^{1}(\Omega)$ such that

$$
\begin{aligned}
\int_{\Omega} \nabla \xi \cdot \nabla w+\left(v\left(x_{1}\right)+v\left(x_{2}\right)+v\left(x_{3}\right)-E\right) \xi w d x & -\int_{\partial \Omega} i \sqrt{E} \xi w d S \\
& =-\int_{\Omega} Q w d x \quad \forall w \in H^{1}(\Omega)
\end{aligned}
$$

The finite element discretization of (41) was then done in a standard fashion using quadratic Lagrange elements on a triangular mesh. The computational domain was divided into sub-domains to have the finite element mesh fit better with the support of the potential $V$ and the constructed function $\chi_{0}$ and the discrepancy $Q$. A relatively uniform mesh was introduced with lengths of triangle edges between 0.15 and 0.48 . With a circular domain of radius 190, the total number of degrees of freedom was 3 million. We used Matlab's solver for large linear systems.

The results are represented by the Fig.2-3, and we think that they are reasonable. 

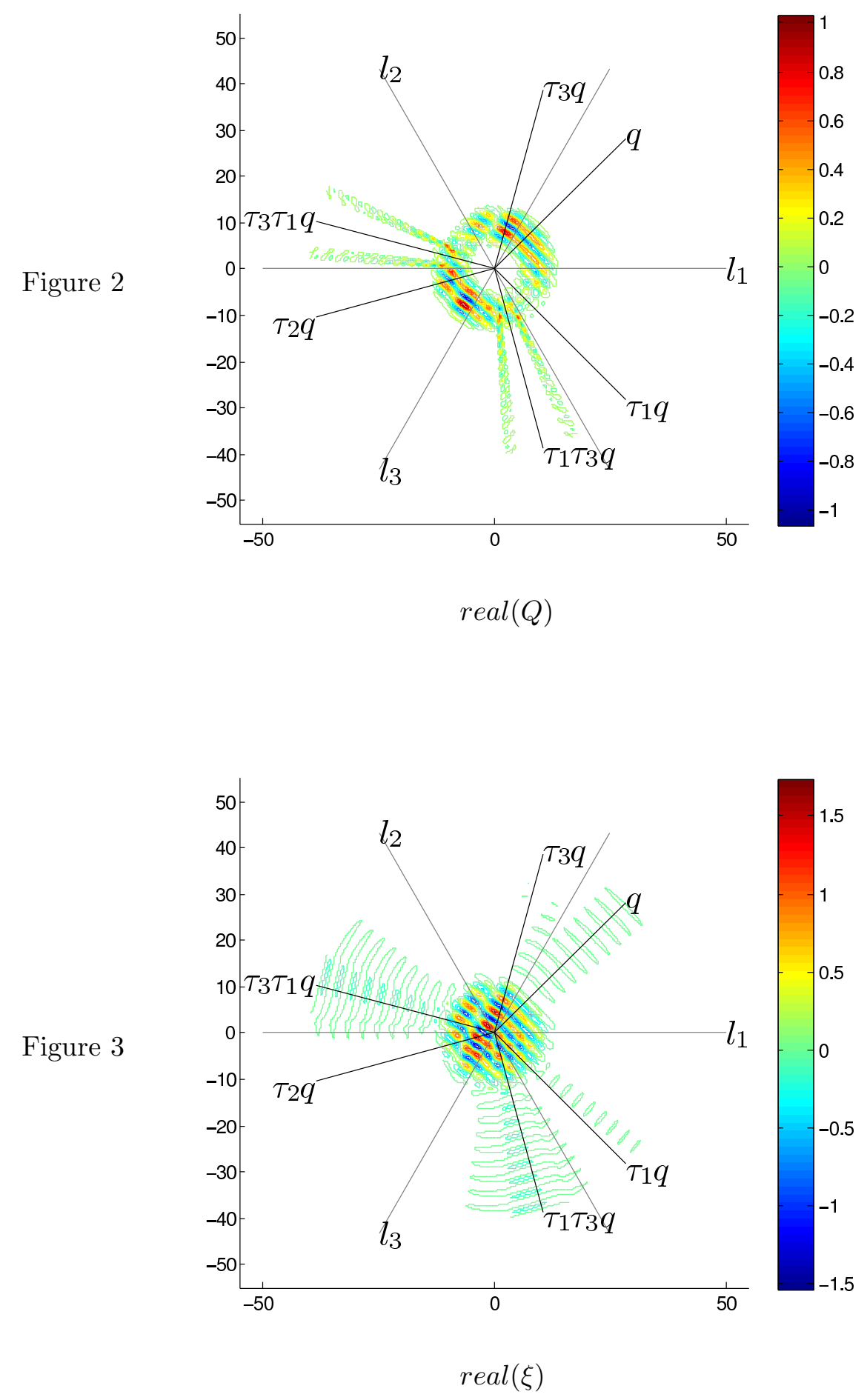

A certain problem was the choice of the radius $R$. To get more precise results it would be better to take bigger $R$, but the bigger $R$ means the harder computations. The criterium of the compromise was connected with the integral form of the radiation conditions. These conditions are:

1)the integral

$$
\int_{S_{R}} d s|\xi(\mathbf{x}, \mathbf{q})|^{2}
$$

over the circle $|\mathbf{x}|=R$ must be bounded for large $R$; 
2) the integral

$$
\int_{S_{R}} d s\left|\left(\frac{\partial}{\partial|\mathbf{x}|}-i \sqrt{E}\right) \xi(\mathbf{x}, \mathbf{q})\right|^{2}
$$

must decrease as $R^{-2}$.

Notice that Fig. 4 shows that the first integral here is asymptotically approaching a constant at sufficiently large $|\mathbf{x}|$, and the second integral is quite small for such $|\mathbf{x}|$, but does not decrease for the present computations with $R=190$.

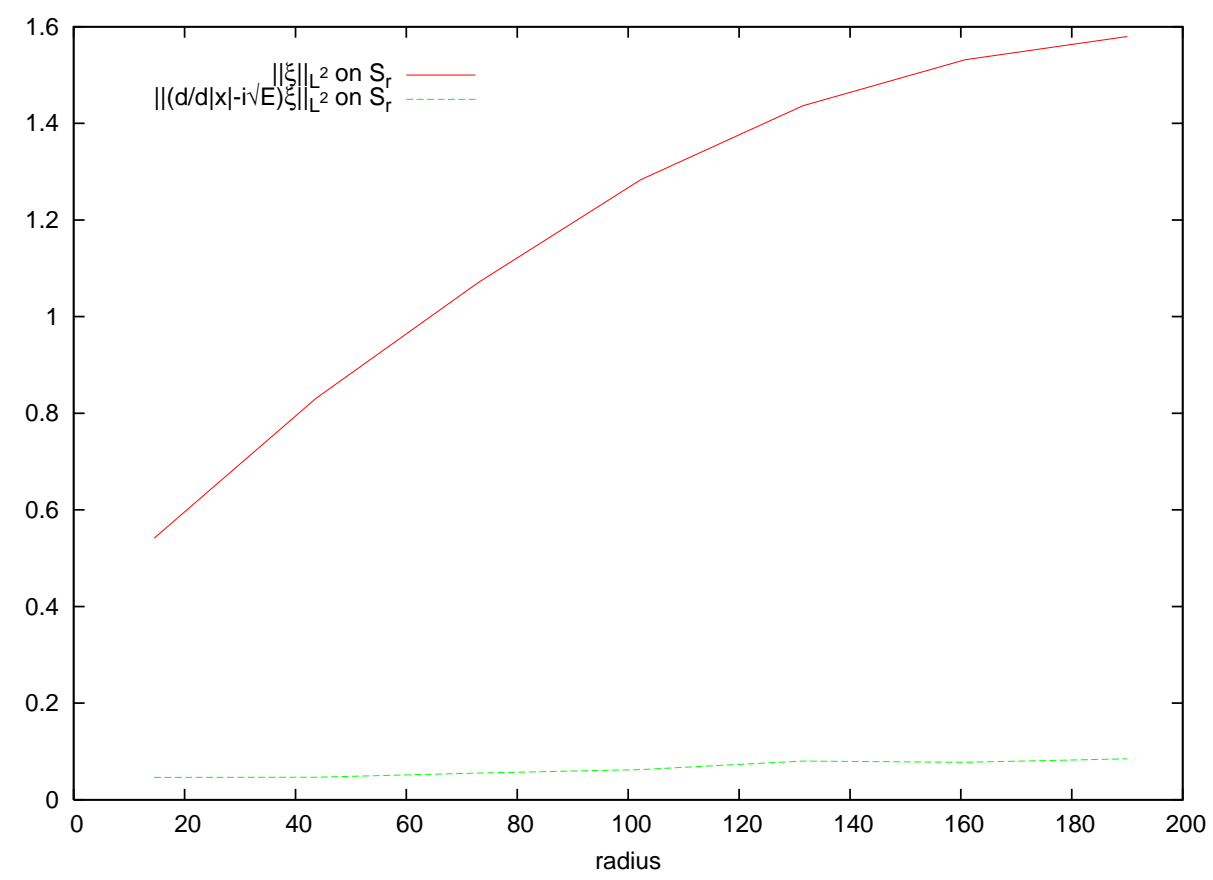

Figure 4

It, probably, means that such radius is not completely sufficient for the final computations. However, the bigger radius would mean the harder computations, so we decided at the moment to restrict the radius of the circle by 190 .

We considered also the corrected boundary condition where the next term of asymptotic behavior of $\xi$ was also taken into account:

$$
\left.\left(\frac{\partial}{\partial r}-i|\mathbf{q}|+\frac{1}{2 r}\right) \xi\right|_{r=R}=0 .
$$

Nevertheless, the correction did not help to stabilize the calculation in smaller domain, as it could be expected. The reason is that the term $\frac{1}{2 R}$ appeared to be very small comparatively with other terms.

To clarify further the situation with the stabilization of $L_{2}$ - norm on the boundary of the disk we should come back to behavior of $\xi$ as a function of the angle at fixed radius $r=R$. 


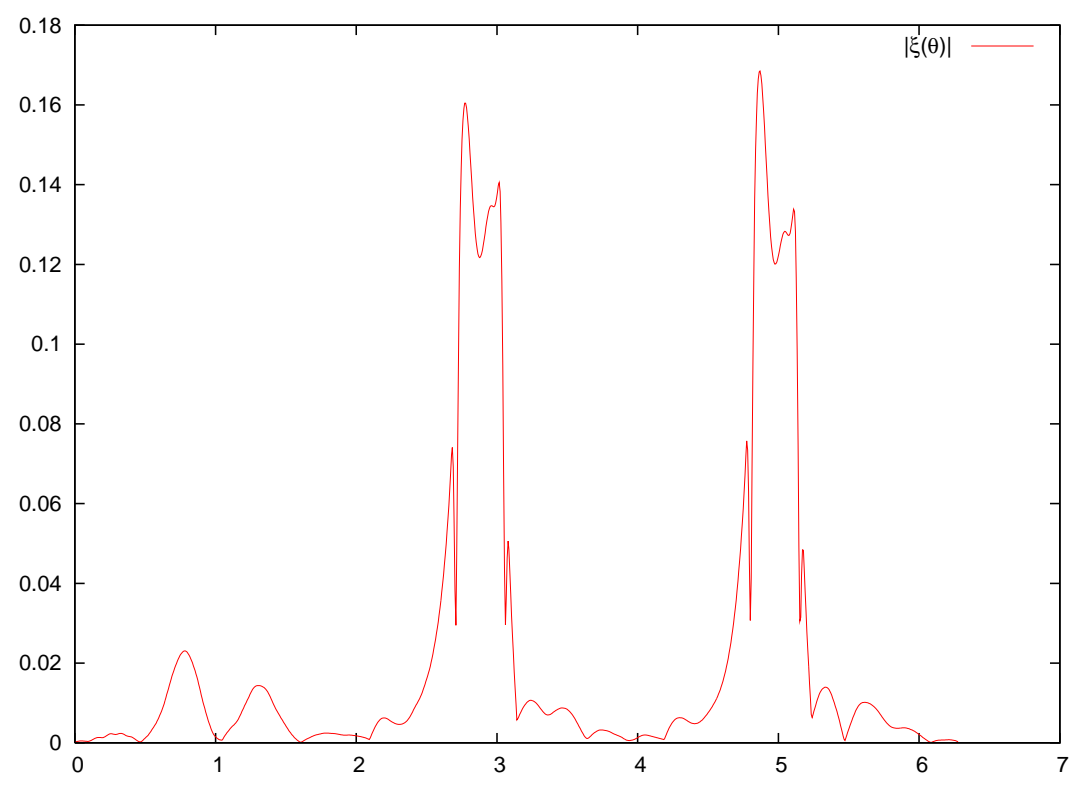

Figure 5

$|\xi(\theta)|$

One can easily see from the Figure that the main contribution to the integral comes from two special directions on the boundaries of shadow and light in sectors $\lambda_{231}$ and $\lambda_{312}$, see Fig.5. Therefore for the stabilization of the whole integrals first of all the contributions to them of two indicated sectors must be stabilized. Namely their stabilization was not completely reached for considered size of configuration domain and requires bigger scale of radii. There are also other computational indications that the behavior of the field in these two sectors is responsible for the (non)stabilization of the $L_{2}$ norms.

On the other hand, we found the right tendency of the behavior of the solution for large $r$ what was the aim of the present calculations.

\section{Acknowledgment}

The authors would like to thank Prof. V.B.Belyaev for the fruitful discussions. The work was partially supported by RFBR grant 08-01-00209.

\section{References}

[1] L. D. Faddeev, Mathematical Aspects of the Three-Body Problem in the Quantum Scattering Theory, Trudy Matematicheskogo Instituta, v.69, (1963) (in Russian), (Israel Program for Scientific Translations, Jerusalem, (1965), (in English)).

[2] V.Enss, Ann.Phys., 119, 117-132, (1979).

[3] J.Derezinski and C.Gerard, J.Math.Phys., v.38(8), pp. 3925-3942, (1997). 
[4] V.S.Buslaev, A.F.Vakulenko, Unitary regularization for the three-body scattering, Vestnik LGU, 13, 22-30, (1977).

[5] E. O. Alt, P. Graßberger, and W. Sandhas, Nucl. Phys. B2, 167, (1967).

[6] R.K. Peterkop, Zh. Eksp. Teor. Fiz, 43, 616 (1962) (in russian) [Sov. Phys. JETP 14, 1377 (1962)].

[7] S.P. Merkuriev, Theor. Math. Phys., 32, 680 (1977); M. Brauner, J.S. Briggs and H.J. Klar, J. Phys. B, 22, 2265 (1989).

[8] E.O. Alt, A.M. Mukhamedzhanov, JETP Lett., 56, 435 (1992), Phys. Rev. A, 47, 2004 (1993); Y.E. Kim, A.L. Zubarev, Phys. Rev. A, 56, 521 (1997).

[9] J.H. Macek, S.Yu. Ovchinnikov, Phys. Rev. A, 54, 1 (1996).

[10] Rudge M R H 1968 Rev. Mod. Phys. 40564

[11] Peterkop R K Theory of Ionization of Atoms by Electron-Impact (Colorado Associated University Press, Boulder, 1977)

[12] Faddeev L D and Merkuriev S P Quantum Scattering Theory for Several Particle Systems (Kluwer, Dordrecht, 1993)

[13] Merkuriev S P 1980 Ann. Phys. (NY) 130395

[14] E. O. Alt and A. M. Mukhamedzhanov, JETP Lett. 56, 435 (1992); Phys. Rev. A 47, 2004 (1993).

[15] E. O. Alt, W. Sandhas, and H. Ziegelmann, Phys. Rev. C 17, 1981 (1978).

[16] Alt E O, Levin S B and Yakovlev S L 2004 Phys. Rev. C 69034002

[17] E. O. Alt and W. Sandhas, in Coulomb Interactions in Nuclear and Atomic FewBody Collisions, edited by F. S. Levin and D. Micha (Plenum, New York 1996), p. 1.

[18] S.Oryu, S.Nishinohara, N.Shiiki, and S.Chiba, Phys. Rev. C 75, 021001(R) (2007).

[19] A.Deltuva, A.C.Fonseca, P.U.Sauer, Phys. Rev. C 71, 054005 (2005).

[20] A. Kievsky, M. Viviani, and S. Rosati, Phys. Rev. C 64, 024002 (2001).

[21] V.B.Belyaev, S.B.Levin, S.L.Yakovlev, J.Phys. B, v.37, 1369-1380, (2004)

[22] V.M.Suslov and B.Vlahovic, Phys.Rev. C, v.69, 044003, (2004)

[23] Rescigno T N, Baertschy M, Isaacs W A and McCurdy C W 1999 Science 286 2474; Baertschy M, Rescigno T N and McCurdy C W 2001 Phys. Rev. A 64 022709; Bray I (2002) Phys. Rev. Lett. 89273201

[24] Buslaev, V. S.; Merkur'ev, S. P., Dokl. Akad. Nauk SSSR 189 269-272 (Russian); translated as Soviet Physics Dokl. 14 (1969) 1055-1057

[25] Buslaev, V. S.; Merkuriev, S. P.; Salikov, S. P. Probl. Mat. Fiz., Leningrad. Univ., Leningrad, 9, (1979), 14-30.

[26] Buslaev, V. S.; Merkuriev, S. P.; Salikov, S. P. (Russian) Zap. Nauchn. Sem. Leningrad. Otdel. Mat. Inst. Steklov. (LOMI) 84, (1979), 16-22.

[27] V.S.Buslaev and S.B.Levin, Amer.Math.Soc.Transl. (2)v.225, pp.55-71, (2008)

[28] A.M.Veselova, Theor.Math.Phys., 35(2), pp. 180-191, (1978).

[29] C. N. Yang, Phys. Rev. Lett. 19, 1312 (1967).

[30] E. Lieb and W. Liniger, Phys. Rev. 130, 1605 (1963). 
[31] J. B. McGuire, J.Math.Phys., 5, 622 (1964).

[32] M. Olshanii, Phys.Rev.Lett., 81, 938 (1998).

[33] N. P. Mehta, B.D. Esry, and C.H.Green, Phys.Rev. A., 76, 022711 (2007).

[34] A Görlitz et al., Phys.Rev.Lett., 87, 130402 (2001).

[35] T. Kinoshita, T. Wenger and D. S. Weiss, Nature (London) 440, 900 (2006).

[36] T. Kinoshita, T. Wenger and D. S. Weiss, Science, 305, 1125 (2004).

[37] J. Esteve et al., Phys.Rev.Lett., 96, 130403 (2006). 